\title{
CONTINUOUS DEPENDENCE OF NONMONOTONIC DISCONTINUOUS DIFFERENTIAL EQUATIONS
}

\author{
DANIEL C. BILES
}

\begin{abstract}
Continuous dependence of solutions for a class of nonmonotonic, discontinuous differential equations is studied. First, a local existence theorem due to $\mathrm{Z}$. Wu is extended to a larger class. Then, a result concerning continuous dependence for this larger class is proven. This employs a type of convergence similar to Gihman's Convergence Criterion, which is defined to be

$$
\text { For all } a, b \text { and } y \quad \lim _{n \rightarrow \infty} \int_{a}^{b} f_{n}(s, y) d s=\int_{a}^{b} f_{\infty}(s, y) d s \text {. }
$$

The significance of Gihman's Convergence Criterion is that for certain classes of differential equations it has been found to be necessary and sufficient for continuous dependence. Finally, examples are presented to motivate and clarify this continuous dependence result.
\end{abstract}

\section{INTRODUCTION}

We consider initial value problems of the form

$$
\begin{aligned}
& u^{\prime}(t)=f(t, u(t)), \quad \text { for } t \in[0, T], \\
& u(0)=x_{0} .
\end{aligned}
$$

A fundamental question one can study concerning (IVP) is that of continuous dependence on $f$, that is, does a small change in $f$ bring about only a small change in $u$ ? Such results are important for at least two reasons in applications. One, $f$ may be the result of an experiment and hence could contain measurement errors. We would like the corresponding solution $u$ to be similar to the "true" solution. Two, given $f$ we may wish to work with some $\tilde{f}$ that is close to $f$, but simpler in some sense (for example, one for which the solution can be obtained by elementary methods). We would like for the solution $\tilde{u}$ (corresponding to $\tilde{f}$ ) to be a good approximation to $u$, the solution of interest. Such continuous dependence results can be stated in terms of sequences, that is,

$$
\text { if } f_{n} \rightarrow f_{\infty} \text {, then } u_{n} \rightarrow u_{\infty},
$$

where $u_{n}$ is the solution corresponding to $f_{n}$, for $n=1,2, \ldots, \infty$. We now consider what types of convergence to use. For the $u$ 's, pointwise or uniform convergence is typically used. For the $f$ 's, these kinds of convergence can also

Received by the editors January 31, 1991.

1991 Mathematics Subject Classification. Primary 34A12; Secondary 34A34.

Key words and phrases. Ordinary differential equations, continuous dependence, local existence. 
be considered. However, several results in the literature, $[1,2,5,6,7,8,9,10$, $11,12,13,14]$ utilize the following:

For each $a, b$ and $y$

(GCC)

$$
\int_{a}^{b} f_{n}(s, y) d s \rightarrow \int_{a}^{b} f_{\infty}(s, y) d s .
$$

We shall call this Gihman's Convergence Criterion, after I. I. Gihman who first used this type of convergence in 1952 [5]. (GCC) is significant for at least two reasons. One, (GCC) is in general weaker than pointwise convergence. (Consider, for example, $f_{n}(s, y) \equiv \sin (n s)$ and $f_{\infty} \equiv 0$.) Two, for certain classes of $f$ 's, some of the papers listed above have found (GCC) to be not only sufficient for convergence of the $u$ 's but also necessary. It is of interest to extend the number of classes of $f$ 's for which (GCC) has been studied.

We now specify the class of problems which is investigated here. Let any $T>0$ be given. We consider $f$ 's which satisfy the following four properties:

(P1) $f$ maps $D \equiv\{(t, x) \mid 0<t<T,-\infty<x<\infty\}$ into $\mathbb{R}$,

(P2) $f$ is bounded on $D$,

(P3) $f$ is Lebesgue measurable in $t$ for each fixed $x \in \mathbb{R}$,

(P4) for each $(t, x) \in D, \lim _{y \rightarrow x+} f(t, y)$ exists and

$$
\limsup _{y \rightarrow x-} f(t, y) \leq f(t, x)=\lim _{y \rightarrow x^{+}} f(t, y) .
$$

In $\S 3$ we shall study continuous dependence of solutions on $f$ for this class. We note that the requirements above are quite weak, since, for example, continuity is not assumed in either variable and $f$ does not need to satisfy a direct monotonicity condition. We note also that condition (P4) does not involve $t$. Also, (P4) can be restated in what may be a more intuitive form, as follows:

(P4) for each fixed $t \in(0, T)$, the function $f(t, \cdot)$ is right-continuous and upper semicontinuous.

Condition (P2) is quite strong, but can probably be replaced by a condition such as:

$\left(\mathbf{P}^{\prime}\right) \quad|f(t, x)| \leq m(t)$ for all $(t, x) \in D$, where $m:(0, T) \rightarrow \mathbb{R}$ is Lebesgue integrable.

This question is not investigated here. We note that continuous dependence was studied for a similar class of $f$ 's in [10], except in that paper $f$ was also required to be nondecreasing in $x$. Thus, the theorem given in $\S 3$, though different in some respects, is in a sense a generalization of that result. In $\S 2$, we consider existence of solutions for this class and finally in $\S 4$ we give examples.

\section{EXISTENCE OF SOLUTIONS}

Before we can discuss continuous dependence of solutions on $f$ for this class, we must verify that solutions actually exist. The literature concerns itself with various definitions of solution (see, for example [ 3 and 4]). We shall seek existence of Carathéodory solutions: $u:[0, T] \rightarrow \mathbb{R}$ is absolutely continuous, $u^{\prime}(t)=f(t, u(t))$ a.e. on $[0, T]$ and $u(0)=x_{0}$. It is well known that Carathéodory solutions may not exist for even simple $f$ 's (for example, consider $f$ defined by $f(t, x)=1$ for $x \leq 0$ and for all $t$, and $f(t, x)=-1$ 
for $x>0$ and for all $t$ ), so existence must indeed be proven. Existence of Carathéodory solutions was demonstrated by $\mathrm{Wu}$ in [15] under slightly stronger conditions than properties (P1), (P2), (P3) and (P4). We use a revision of Wu's argument to weaken his original hypotheses. The following proof also contains an additional parameter $\varepsilon$. This parameter is unnecessary for the proof of existence (i.e., $\varepsilon=0$ can be used), but will be useful in the proof of continuous dependence in the next section.

Theorem 1. Let any $T>0$ and $x_{0} \in \mathbb{R}$ be given. Let $f$ satisfy properties ( $\left.\mathrm{P} 1\right)$, (P?), (P3) and (P4).

Then, a Carathéodory solution of (IVP) exists on $[0, T]$ and among these solutions there is a greatest one.

Proof. For each $\varepsilon \geq 0$, we shall construct a nonincreasing sequence of continuous functions $x_{1, \varepsilon}, x_{2, \varepsilon}, \ldots$ which converge to some continuous function $u_{\varepsilon}$. We shall then show that $u_{0}$ is the greatest solution to (IVP).

Fix any $\varepsilon \geq 0$. For each $n \in \mathbb{N}$, we shall choose a partition of $[0, T]$,

$$
0=\sigma_{0}^{(n, \varepsilon)}<\sigma_{1}^{(n, \varepsilon)}<\cdots<\sigma_{l_{n, \varepsilon}}^{(n, \varepsilon)}=T
$$

with $\sigma_{i+1}^{(n, \varepsilon)}-\sigma_{i}^{(n, \varepsilon)} \leq 2^{-n} T$, and a function $x_{n, \varepsilon}:[0, T] \rightarrow \mathbb{R}$ by a procedure discussed below. We define

$$
B_{k, n, \varepsilon}(s)=\left\{x:\left|x-x_{n, \varepsilon}\left(\sigma_{k}^{(n, \varepsilon)}\right)\right| \leq 2 M\left(s-\sigma_{k}^{(n, \varepsilon)}\right)+\varepsilon\right\},
$$

where $M$ is any number such that $|f(t, x)| \leq M$ for all $(t, x) \in D$. We also denote

$$
g_{k, n, \varepsilon}(s)=\sup \left\{f(s, x): x \in B_{k, n, \varepsilon}(s)\right\}
$$

and

$$
a_{n, \varepsilon}(s)=g_{k, n, \varepsilon}(s) \text { for } \sigma_{k}^{(n, \varepsilon)} \leq s<\sigma_{k+1}^{(n, \varepsilon)} .
$$

We shall define $x_{n, \varepsilon}:[0, T] \rightarrow \mathbb{R}$ by

$$
\begin{aligned}
& x_{n, \varepsilon}(0)=x_{0}, \\
& x_{n, \varepsilon}(t)=x_{n, \varepsilon}\left(\sigma_{k}^{(n, \varepsilon)}\right)+\int_{\sigma_{k}^{(n, \varepsilon)}}^{t} g_{k, n, \varepsilon}(s) d s, \quad \text { for } t \in\left[\sigma_{k}^{(n, \varepsilon)}, \sigma_{k+1}^{(n, \varepsilon)}\right] .
\end{aligned}
$$

Note that we may also write $x_{n, \varepsilon}$ as $x_{n, \varepsilon}(t)=x_{0}+\int_{0}^{t} a_{n, \varepsilon}(s) d s$. Note also that it suffices to choose the $\sigma_{k}^{(n, \varepsilon)}$ 's in order to specify $x_{n, \varepsilon}$.

For $n=1$, we partition [0,T] by letting $\sigma_{k}^{(1, \varepsilon)}-\sigma_{k-1}^{(1, \varepsilon)}=T / 2$, for $k=1,2$. Thus, we have defined $x_{1, \varepsilon}$ over all of $[0, T]$.

Now let $n \in \mathbb{N}, n \neq 1$, be given. Assume $x_{j, \varepsilon}$ and the $\sigma_{k}^{(j, \varepsilon)}$,s have already been chosen, for all $j<n$. We also assume $x_{1, \varepsilon}(t) \geq x_{2, \varepsilon}(t) \geq \cdots \geq x_{n-1, \varepsilon}(t)$ for each $t \in[0, T]$. Let any $i \in\{0,1,2, \ldots\}$ be given, and suppose that $\sigma_{0}^{(n, \varepsilon)}, \sigma_{1}^{(n, \varepsilon)}, \ldots, \sigma_{i}^{(n, \varepsilon)}$ have been chosen, with $0=\sigma_{0}^{(n, \varepsilon)}<\sigma_{1}^{(n, \varepsilon)}<\cdots<$ $\sigma_{i}^{(n, \varepsilon)}<T$ and that $x_{n, \varepsilon}$ has been defined on $\left[0, \sigma_{i}^{(n, \varepsilon)}\right]$ with $x_{n, \varepsilon} \leq x_{n-1, \varepsilon}$ on that interval. We now define a function $\widetilde{x_{n, \varepsilon}}$ on $\left[\sigma_{i}^{(n, \varepsilon)}, T\right]$ as follows:

$$
\widetilde{x_{n, \varepsilon}}(t)=x_{n, \varepsilon}\left(\sigma_{i}^{(n, \varepsilon)}\right)+\int_{\sigma_{i}^{(n, \varepsilon)}}^{t} g_{i, n, \varepsilon}(s) d s .
$$


Note that $\widetilde{x_{n, \varepsilon}}\left(\sigma_{i}^{(n, \varepsilon)}\right)=x_{n, \varepsilon}\left(\sigma_{i}^{(n, \varepsilon)}\right) \leq x_{n-1, \varepsilon}\left(\sigma_{i}^{(n, \varepsilon)}\right)$. We choose $\sigma_{i+1}^{(n, \varepsilon)}$ in one of several ways, depending on how $\widetilde{x_{n, \varepsilon}}\left(\sigma_{i}^{(n, \varepsilon)}\right)$ is related to $x_{n-1, \varepsilon}\left(\sigma_{i}^{(n, \varepsilon)}\right)$. In what follows, we shall use $\tau \equiv \min \left\{T, \sigma_{i}^{(n, \varepsilon)}+2^{-n} T\right\}$.

Case One. $\widetilde{x_{n, \varepsilon}}\left(\sigma_{i}^{(n, \varepsilon)}\right)<x_{n-1, \varepsilon}\left(\sigma_{i}^{(n, \varepsilon)}\right)$.

Subcase a. $\widetilde{x_{n, \varepsilon}}(t) \leq x_{n-1, \varepsilon}(t)$ for all $t \in\left[\sigma_{i}^{(n, \varepsilon)}, \tau\right]$. In this subcase, let $\sigma_{i+1}^{(n, \varepsilon)}=\tau$ and define $x_{n, \varepsilon}(t)=\widetilde{x_{n, \varepsilon}}(t)$ on $\left[\sigma_{i}^{(n, \varepsilon)}, \sigma_{i+1}^{(n, \varepsilon)}\right]$. Obviously, we have $x_{n, \varepsilon}(t) \leq x_{n-1, \varepsilon}(t)$ on $\left[\sigma_{i}^{(n, \varepsilon)}, \sigma_{i+1}^{(n, \varepsilon)}\right]$.

Subcase b. $\widetilde{x_{n, \varepsilon}}(t)>x_{n-1, \varepsilon}(t)$ for some $t \in\left(\sigma_{i}^{(n, \varepsilon)}, \tau\right)$. Define $\sigma_{i+1}^{(n, \varepsilon)}=$ $\inf \left\{t \in\left(\sigma_{i}^{(n, \varepsilon)}, \tau\right) \mid \widetilde{x_{n, \varepsilon}}(t)>x_{n-1, \varepsilon}(t)\right\}$. Then, $\widetilde{x_{n, \varepsilon}}\left(\sigma_{i+1}^{(n, \varepsilon)}\right)=x_{n-1, \varepsilon}\left(\sigma_{i+1}^{(n, \varepsilon)}\right)$ and $\widetilde{x_{n, \varepsilon}}(t) \leq x_{n-1, \varepsilon}(t)$ for all $t \in\left[\sigma_{i}^{(n, \varepsilon)}, \sigma_{i+1}^{(n, \varepsilon)}\right]$. Define $x_{n, \varepsilon}(t)=\widetilde{x_{n, \varepsilon}}(t)$ on $\sigma_{i}^{(n, \varepsilon)} \leq t \leq \sigma_{i+1}^{(n, \varepsilon)}$. Obviously, $x_{n, \varepsilon}(t) \leq x_{n-1, \varepsilon}(t)$ on that interval.

Case Two. $\widetilde{x_{n, \varepsilon}}\left(\sigma_{i}^{(n, \varepsilon)}\right)=x_{n-1, \varepsilon}\left(\sigma_{i}^{(n, \varepsilon)}\right)$. In this case, define

$$
\sigma_{i+1}^{(n, \varepsilon)}=\min \left\{\tau, \min _{k}\left\{\sigma_{k}^{(n-1, \varepsilon)}: \sigma_{k}^{(n-1, \varepsilon)}>\sigma_{i}^{(n, \varepsilon)}\right\}\right\} \text {. }
$$

Note that $\left[\sigma_{i}^{(n, \varepsilon)}, \sigma_{i+1}^{(n, \varepsilon)}\right] \subseteq\left[\sigma_{j}^{(n-1, \varepsilon)}, \sigma_{j+1}^{(n-1, \varepsilon)}\right]$ for some $j$. Define $x_{n, \varepsilon}(t)=$ $\widetilde{x_{n, \varepsilon}}(t)$ on $\left[\sigma_{i}^{(n, \varepsilon)}, \sigma_{i+1}^{(n, \varepsilon)}\right]$. Fix any $t \in\left[\sigma_{i}^{(n, \varepsilon)}, \sigma_{i+1}^{(n, \varepsilon)}\right]$. We claim $x_{n, \varepsilon}(t) \leq$ $x_{n-1, \varepsilon}(t)$. Choose $j$ as above. Recall that

$$
x_{n, \varepsilon}(t)=x_{n, \varepsilon}\left(\sigma_{i}^{(n, \varepsilon)}\right)+\int_{\sigma_{i}^{(n, \ell)}}^{t} \sup \left\{f(s, x): x \in B_{i, n, \varepsilon}(s)\right\} d s
$$

and

$$
x_{n-1, \varepsilon}(t)=x_{n-1, \varepsilon}\left(\sigma_{i}^{(n, \varepsilon)}\right)+\int_{\sigma_{i}^{(n, \ell)}}^{t} \sup \left\{f(s, x): x \in B_{j, n-1, \varepsilon}(s)\right\} d s .
$$

Thus, it suffices to show $B_{i, n, \varepsilon}(s) \subseteq B_{j, n-1, \varepsilon}(s)$ for $s \in\left[\sigma_{i}^{(n, \varepsilon)}, t\right]$. Note that $\sigma_{j}^{(n-1, \varepsilon)} \leq \sigma_{i}^{(n, \varepsilon)} \leq t \leq \sigma_{i+1}^{(n, \varepsilon)} \leq \sigma_{j+1}^{(n-1, \varepsilon)}$. Let $x \in B_{i, n, \varepsilon}(s)$. We then have $\left|x-x_{n, \varepsilon}\left(\sigma_{i}^{(n, \varepsilon)}\right)\right| \leq 2 M\left(s-\sigma_{i}^{(n, \varepsilon)}\right)+\varepsilon$ and so

$$
\begin{aligned}
\mid x- & x_{n-1, \varepsilon}\left(\sigma_{j}^{(n-1, \varepsilon)}\right) \mid \\
& \leq\left|x-x_{n, \varepsilon}\left(\sigma_{i}^{(n, \varepsilon)}\right)\right|+\left|x_{n, \varepsilon}\left(\sigma_{i}^{(n, \varepsilon)}\right)-x_{n-1, \varepsilon}\left(\sigma_{j}^{(n-1, \varepsilon)}\right)\right| \\
& \leq 2 M\left(s-\sigma_{i}^{(n, \varepsilon)}\right)+\varepsilon+\left|\int_{\sigma_{j}^{(n-1, \varepsilon)}}^{\sigma_{i}^{(n, \varepsilon)}} g_{j, n-1, \varepsilon}(s) d s\right| \\
& \leq 2 M\left(s-\sigma_{i}^{(n, \varepsilon)}\right)+\varepsilon+M\left(\sigma_{i}^{(n, \varepsilon)}-\sigma_{j}^{(n-1, \varepsilon)}\right) \\
& =2 M s+\varepsilon-2 M\left(\frac{\sigma_{i}^{(n, \varepsilon)}+\sigma_{j}^{(n-1, \varepsilon)}}{2}\right) \\
& \leq 2 M s+\varepsilon-2 M \sigma_{j}^{(n-1, \varepsilon)} \\
& =2 M\left(s-\sigma_{j}^{(n-1, \varepsilon)}\right)+\varepsilon .
\end{aligned}
$$

That is, $x \in B_{j, n-1, \varepsilon}(s)$. We therefore have $x_{n, \varepsilon}(t) \leq x_{n-1, \varepsilon}(t)$ on $\left[\sigma_{i}^{(n, \varepsilon)}\right.$, $\left.\sigma_{i+1}^{(n, \varepsilon)}\right]$. Note that $\sigma_{i+1}^{(n, \varepsilon)}-\sigma_{i}^{(n, \varepsilon)} \leq 2^{-n} T$. 
We continue this process along the interval $[0, T]$ until we reach some $k$ such that $\sigma_{k}^{(n, \varepsilon)}=T$. We must verify that this actually happens. Clearly, the sequence $\sigma_{1}^{(n, \varepsilon)}, \sigma_{2}^{(n, \varepsilon)}, \ldots$ is strictly increasing, so we need only prove the following.

Claim. For each fixed $n \in \mathbb{N},\left\{\sigma_{i}^{(n, \varepsilon)}\right\}$ does not converge to some number less than or equal to $T$.

Proof of Claim. We shall handle this inductively. For $n=1$, we have the desired situation, since $\sigma_{0}^{(1, \varepsilon)}=0, \sigma_{1}^{(1, \varepsilon)}=T / 2$, and $\sigma_{2}^{(1, \varepsilon)}=T$. We now assume the claim holds for $n-1$. We thus have $\sigma_{j+1}^{(n-1, \varepsilon)}-\sigma_{j}^{(n-1, \varepsilon)}>$ some positive constant $c_{n-1}$ for all $j$. Let any $i \in\{0,1,2, \ldots\}$ be given. It suffices to show $\sigma_{i+4}^{(n, \varepsilon)}-\sigma_{i}^{(n, \varepsilon)}>$ some positive constant $d_{n}$. For convenience, in Case Two if $\sigma_{m+1}^{(n, \varepsilon)}$ is chosen to be $\tau$ for some $m$ in question, we say $\sigma_{m}^{(n, \varepsilon)}$ satisfies case (2a). If $\sigma_{m+1}^{(n, \varepsilon)}$ is chosen to be $\min _{k}\left\{\sigma_{k}^{(n-1, \varepsilon)}: \sigma_{k}^{(n-1, \varepsilon)}>\sigma_{m}^{(n, \varepsilon)}\right\}$ for some $m$ in question, we say $\sigma_{m}^{(n, \varepsilon)}$ satisfies case $(2 \mathrm{~b})$.

Possibility One. If $\sigma_{i}^{(n, \varepsilon)}$ satisfies case (1a) (that is, Case One, subcase (a), then $\sigma_{i+4}^{(n, \varepsilon)}-\sigma_{i}^{(n, \varepsilon)}>\sigma_{i+1}^{(n, \varepsilon)}-\sigma_{i}^{(n, \varepsilon)}=2^{-n} T$.

Possibility Two. If $\sigma_{i}^{(n, \varepsilon)}$ satisfies case (2a), then $\sigma_{i+4}^{(n, \varepsilon)}-\sigma_{i}^{(n, \varepsilon)}>\sigma_{i+1}^{(n, \varepsilon)}-$ $\sigma_{i}^{(n, \varepsilon)}=2^{-n} T$.

To make the remaining possibilities simpler, we shall assume $\sigma_{m}^{(n, \varepsilon)}$ does not satisfy case (1a) or case $(2 \mathrm{a})$ for $m=i+1, i+2$, or $i+3$, since it is obvious we can handle those situations as above. Tine only possibilities which still need to be studied are $\sigma_{i}^{(n, \varepsilon)}$ satisfying case (1b) and $\sigma_{i}^{(n, \varepsilon)}$ satisfying case $(2 b)$. Note that case $(1 b)$ cannot occur at two consecutive points $\sigma_{m}^{(n, \varepsilon)}$ and $\sigma_{m+1}^{(n, \varepsilon)}$, since $\sigma_{m}^{(n, \varepsilon)}$ satisfying (1b) implies $x_{n}\left(\sigma_{m}^{(n, \varepsilon)}\right)<x_{n-1}\left(\sigma_{m}^{(n, \varepsilon)}\right)$ and $x_{n}\left(\sigma_{m+1}^{(m, \varepsilon)}\right)=x_{n-1}\left(\sigma_{m+1}^{(n, \varepsilon)}\right)$.

Possibility Three. Suppose $\sigma_{i}^{(n, \varepsilon)}$ satisfies case (2b). Then, $\sigma_{i+1}^{(n, \varepsilon)}=\sigma_{j}^{(n-1, \varepsilon)}$ for some $j \in \mathbb{N}$. Suppose for now that $\sigma_{i+1}^{(n, \varepsilon)}$ also satisfies case (2b). Then, $\sigma_{i+2}^{(n, \varepsilon)}=\sigma_{j+1}^{(n-1, \varepsilon)}$. We then have $\sigma_{i+4}^{(n-1, \varepsilon)}-\sigma_{i}^{(n, \varepsilon)}>\sigma_{i+2}^{(n, \varepsilon)}-\sigma_{i+1}^{(n, \varepsilon)}=\sigma_{j+1}^{(n-1, \varepsilon)}-$ $\sigma_{j}^{(n-1, \varepsilon)}>c_{n-1}$. The only other possibility to check is $\sigma_{i+1}^{(n, \varepsilon)}$ satisfying case (1b). Then, $\sigma_{i+2}^{(n, \varepsilon)}$ must satisfy case $(2 \mathrm{~b})$, that is, $\sigma_{i+3}^{(n, \varepsilon)}=\sigma_{l}^{(n-1, \varepsilon)}$ for some $l>j$. We then have $\sigma_{i+4}^{(n, \varepsilon)}-\sigma_{i}^{(n, \varepsilon)}>\sigma_{i+3}^{(n, \varepsilon)}-\sigma_{i+1}^{(n, \varepsilon)}=\sigma_{l}^{(n-1, \varepsilon)}-\sigma_{j}^{(n-1, \varepsilon)}>c_{n-1}$. Possibility Four. Suppose $\sigma_{i}^{(n, \varepsilon)}$ satisfies case (1b). Then, $\sigma_{i+1}^{(n, \varepsilon)}$ must satisfy case $(2 \mathrm{~b})$. We note that in Possibility Three we actually showed that $\sigma_{i+3}^{(n, \varepsilon)}-$ $\sigma_{i}^{(n, \varepsilon)}>c_{n-1}$. Hence, we can repeat the argument there, replacing $i$ by $i+1$. We then have $\sigma_{i+4}^{(n, \varepsilon)}-\sigma_{i}^{(n, \varepsilon)}>\sigma_{i+4}^{(n, \varepsilon)}-\sigma_{i+1}^{(n, \varepsilon)}>c_{n-1}$.

In all four possibilities, we have $\sigma_{i+4}^{(n, \varepsilon)}-\sigma_{i}^{(n, \varepsilon)}>\min \left\{c_{n-1}, 2^{-n} T\right\} \equiv d_{n}>0$. Therefore, we have established the claim.

We have thus constructed the partition $\left\{\sigma_{i}^{(n, \varepsilon)}\right\}$ which determines the function $x_{n, \varepsilon}$. Note that we have $x_{1, \varepsilon}(t) \geq x_{2, \varepsilon}(t) \geq \cdots \geq x_{n, \varepsilon}(t) \geq \cdots$ for all $t \in[0, T]$, as desired. 
We shall now show the hypotheses to the Ascoli-Arzela lemma are satisfied. We first note that $\left\{x_{n, \varepsilon}\right\}$ is uniformly bounded, since

$$
\left|x_{n, \varepsilon}(t)\right|=\left|x_{0}+\int_{0}^{t} a_{n, \varepsilon}(s) d s\right| \leq\left|x_{0}\right|+\int_{0}^{t}\left|a_{n, \varepsilon}(s)\right| d s \leq\left|x_{0}\right|+M T,
$$

which is independent of $n$ and $t$. Also, $\left|x_{n, \varepsilon}^{\prime}(t)\right|=\left|a_{n, \varepsilon}(t)\right| \leq M$ for almost all $t \in[0, T]$, hence $\left\{x_{n, \varepsilon}\right\}$ is equicontinuous. Applying the Ascoli-Arzela lemma, there is some subsequence of $\left\{x_{n, \varepsilon}\right\}$ that converges uniformly to some continuous function $u_{\varepsilon}$. Because of the monotonicity of $\left\{x_{n, \varepsilon}\right\}$, the entire sequence actually converges uniformly to $u_{\varepsilon}$.

Consider the special case $\varepsilon=0$. We propose to show $u_{0}$ is a solution to (IVP). Fix any $t \in(0, T)$. We shall show $\lim _{n \rightarrow \infty} a_{n, 0}(t)=f\left(t, u_{0}(t)\right)$. For each $n$, there exists some $k$ so that $t \in\left[\sigma_{k}^{(n, 0)}, \sigma_{k+1}^{(n, 0)}\right)$. We claim that $x_{n, 0}(t) \in B_{k, n, 0}(t)$. To see this, note that

$$
\begin{aligned}
& \left|x_{n, 0}(t)-x_{n, 0}\left(\sigma_{k}^{(n, 0)}\right)\right| \\
& \quad=\left|x_{0}+\int_{0}^{t} a_{n, 0}(s) d s-x_{0}-\int_{0}^{\sigma_{k}^{(n, 0)}} a_{n, 0}(s) d s\right| \\
& \quad \leq \int_{\sigma_{k}^{(n, 0)}}^{t}\left|a_{n, 0}(s)\right| d s \leq M\left(t-\sigma_{k}^{(n, 0)}\right) \leq 2 M\left(t-\sigma_{k}^{(n, 0)}\right)
\end{aligned}
$$

hence, $x_{n, 0}(t) \in B_{k, n, 0}(t)$. We therefore have

$$
a_{n, 0}(t)=g_{k, n, 0}(t) \equiv \sup \left\{f(t, x): x \in B_{k, n, 0}(t)\right\} \geq f\left(t, x_{n, 0}(t)\right),
$$

and thus

$$
\begin{aligned}
\liminf _{n \rightarrow \infty} a_{n, 0}(t) & \geq \liminf _{n \rightarrow \infty} f\left(t, x_{n}, 0(t)\right) \\
& =\lim _{n \rightarrow \infty} f\left(t, x_{n, 0}(t)\right)=f\left(t, u_{0}(t)\right),
\end{aligned}
$$

since $x_{n, 0}(t) \downarrow u_{0}(t)$ and $f$ is right-continuous in its second variable.

Now temporarily fix any $n \in \mathbb{N}$. Choose $k$ as before, i.e., so that $t \in$ $\left[\sigma_{k}^{(n, 0)}, \sigma_{k+1}^{(n, 0)}\right)$. Again, we have $x_{n, 0}(t) \in B_{k, n, 0}(t)$. Since $a_{n, 0}(t)=g_{k, n, 0}(t)$ $\equiv \sup \left\{f(t, x): x \in B_{k, n, 0}(t)\right\}$, there exists some $\hat{x}_{n} \in B_{k, n, 0}(t)$ such that

$$
a_{n, 0}(t)-2^{-n}<f\left(t, \hat{x}_{n}\right) \leq a_{n, 0}(t) .
$$

Therefore,

$$
\limsup _{n \rightarrow \infty} a_{n, 0}(t)=\limsup _{n \rightarrow \infty} f\left(t, \hat{x}_{n}\right) .
$$

Note that, since $\hat{x}_{n}$ and $x_{n, 0}(t)$ both lie in $B_{k, n, 0}(t)$,

$$
\left|\hat{x}_{n}-x_{n, 0}(t)\right| \leq 2\left[2 M\left(t-\sigma_{k}^{(n, 0)}\right)\right] \leq 4 M T / 2^{n} .
$$

Since the right-hand side of the inequalities approaches 0 as $n \rightarrow \infty$, we have

$$
\lim _{n \rightarrow \infty} \hat{x}_{n}=\lim _{n \rightarrow \infty} x_{n, 0}(t)=u_{0}(t) .
$$

Because of our assumption (P4), we have

$$
\limsup _{n \rightarrow \infty} a_{n, 0}(t) \stackrel{(1)}{=} \limsup _{n \rightarrow \infty} f\left(t, \hat{x}_{n}\right) \leq f\left(t, u_{0}(t)\right) .
$$


Therefore,

$$
\liminf _{n \rightarrow \infty} a_{n, 0}(t) \geq f\left(t, u_{0}(t)\right) \geq \limsup _{n \rightarrow \infty} a_{n, 0}(t),
$$

and thus $\lim _{n \rightarrow \infty} a_{n, 0}(t)=f\left(t, u_{0}(t)\right)$.

We thus have, by the Lebesgue Dominated Convergence Theorem,

$$
\begin{aligned}
u_{0}(t) & =\lim _{n \rightarrow \infty} x_{n, 0}(t)=\lim _{n \rightarrow \infty}\left[x_{0}+\int_{0}^{t} a_{n, 0}(s) d s\right] \\
& =x_{0}+\int_{0}^{t} \lim _{n \rightarrow \infty} a_{n, 0}(s) d s=x_{0}+\int_{0}^{t} f\left(s, u_{0}(s)\right) d s .
\end{aligned}
$$

That is, $u_{0}$ is a solution of (IVP).

It remains to show that $u_{0}$ is actually the greatest solution of (IVP). That is, we shall show $u_{0}(t) \geq \bar{u}(t)$ for all $t \in[0, T]$, where $\bar{u}$ is any solution of (IVP). Suppose $u_{0}$ is not the greatest solution. Then, there exists some $\bar{t} \in(0, T)$ and a solution $\bar{u}$ such that $u_{0}(\bar{t})<\bar{u}(\bar{t})$. Since $x_{n, 0}(\bar{t}) \downarrow u_{0}(\bar{t})$, there exists some $N \in \mathbb{N}$ such that $x_{N, 0}(\bar{t})<\bar{u}(\bar{t})$. Let $a \equiv \inf \left\{t \in[0, \bar{t}): x_{N, 0}<\bar{u}\right.$ on $(t, \bar{t}]\}$. Note that $x_{N, 0}(a)=\bar{u}(a)$, since both $x_{N, 0}$ and $\bar{u}$ are continuous. Note also that there exists some subinterval $\left[\sigma_{k}^{(N, 0)}, \sigma_{k+1}^{(N, 0)}\right)$ and some $b>a$ such that $[a, b] \subseteq\left[\sigma_{k}^{(N, 0)}, \sigma_{k+1}^{(N, 0)}\right)$ and $x_{N, 0}(t)<\bar{u}(t)$ for all $t \in(a, b]$. We claim $\bar{u}(s) \in B_{k, N, 0}(s)$ for all $s \in[a, b]$. Keeping in mind that $\sigma_{k}^{(N, 0)} \leq a \leq$ $s \leq b<\sigma_{k+1}^{(N, 0)}$, we have

$$
\begin{aligned}
\mid \bar{u}(s) & -x_{N, 0}\left(\sigma_{k}^{(N, 0)}\right) \mid \\
& =\left|\bar{u}(a)+\int_{a}^{s} f(r, \bar{u}(r)) d r-x_{N, 0}(a)+\int_{\sigma_{k}^{(N, 0)}}^{a} a_{N, 0}(s) d s\right| \\
& =\left|\int_{a}^{s} f(r, \bar{u}(r)) d r+\int_{\sigma_{k}(N, 0)}^{a} a_{N, 0}(s) d s\right| \\
& \leq M(s-a)+M\left(a-\sigma_{k}^{(N, 0)}\right) \\
& \leq 2 M\left(s-\sigma_{k}^{(N, 0)}\right) .
\end{aligned}
$$

Thus, $\bar{u}(s) \in B_{k, N, 0}(s)$, and so $f(s, \bar{u}(s)) \leq g_{k, N, 0}(s)$. Therefore, for any $t \in(a, b]$, we have

$$
\begin{aligned}
0 & <\bar{u}(t)-x_{N, 0}(t)=[\bar{u}(t)-\bar{u}(a)]-\left[x_{N, 0}(t)-x_{N, 0}(a)\right] \\
& =\int_{a}^{t} f(s, \bar{u}(s)) d s-\int_{a}^{t} g_{k, N, 0}(s) d s \leq 0
\end{aligned}
$$

a contradiction. Hence, $u_{0}$ is the greatest solution to (IVP).

(The referee pointed out an interesting characterization of the $u_{\varepsilon}$ 's, constructed in the above proof and utilized in the proof of Theorem 2. He was able to prove that if we define $f_{\varepsilon}(t, x)=\sup \{f(t, w):|w-x| \leq \varepsilon\}$, then $f_{\varepsilon}$ satisfies properties (P1)-(P4) and hence (by Theorem 1$)$ the initial value problem $v^{\prime}(t)=f_{\varepsilon}(t, v(t)), v(0)=x_{0}$ has a greatest solution. He then showed that greatest solution was in fact $u_{\varepsilon}$.) 


\section{CONTINUOUS DEPENDENCE}

The main result of this section is Theorem 2, a continuous dependence result for initial value problems of the type introduced in the previous sections. We first present two lemmas needed to prove that theorem.

Lemma 1. Let $f(t, x): \mathbb{R} \times \mathbb{R} \rightarrow \mathbb{R}$ be Lebesgue measurable in $t$ for fixed $x$, right-continuous in $x$ for fixed $t$, and bounded. Let any $c, d \in \mathbb{R}$ with $c \leq d$ be given. Let $\mathscr{F} \equiv\{v:[c, d] \rightarrow \mathbb{R} \mid v$ is Lebesgue measurable $\}$.

Then,

$$
\sup _{v \in \mathscr{I}} \int_{c}^{d} f(s, v(s)) d s=\int_{c}^{d} \sup _{y \in \mathbb{R}} f(s, y) d s .
$$

Proof. We shall first verify that the left-hand and right-hand sides exist and are finite. Recalling that the composition of a Borel measurable function and a Lebesgue measurable function, in the appropriate order, is Lebesgue measurable, and noting also that $f$ is bounded, it follows that $\int_{c}^{d} f(s, v(s)) d s$ exists and is finite for each $v$. In fact, it is bounded uniformly in $v$. Hence, $\sup _{v \in \mathscr{F}} \int_{c}^{d} f(s, v(s)) d s$ exists and is finite.

Let $E:[c, d] \rightarrow \mathbb{R}$ be defined by $E(s)=\sup _{y \in \mathbb{R}} f(s, y)$. Let $\left\{y_{j}\right\}_{j=1}^{\infty}$ be any countable dense set in $\mathbb{R}$ (for instance, the rationals). It follows from the right-continuity hypothesis that

$$
E(s)=\sup _{j} f\left(s, y_{j}\right) \quad \text { for each } s \in[c, d] .
$$

Claim. $\int_{c}^{d} \sup _{y \in \mathbf{R}} f(s, y) d s$ exists and is finite.

Proof of Claim. For fixed $y \in \mathbb{R}, f(\cdot, y):[c, d] \rightarrow \mathbb{R}$ is bounded and Lebesgue measurable. It is well known that the sup of a countable number of Lebesgue measurable functions which are bounded uniformly is a bounded Lebesgue measurable function. Hence, $\sup _{y \in \mathbf{R}} f(s, y) \stackrel{(2)}{=} \sup _{j} f\left(s, y_{j}\right)$ is bounded and Lebesgue measurable, so $\int_{c}^{d} \sup _{y \in \mathbf{R}} f(s, y) d s$ exists and is finite.

We now proceed to verify the desired equation. Fix any $v_{0} \in \mathscr{F}$. We then have, for each $s \in[c, d]$

$$
f\left(s, v_{0}(s)\right) \leq \sup _{y \in \mathbf{R}} f(s, y),
$$

since $v_{0}(s) \in \mathbb{R}$. Hence,

$$
\int_{c}^{d} f\left(s, v_{0}(s)\right) d s \leq \int_{c}^{d} \sup _{y \in \mathbb{R}} f(s, y) d s,
$$

and therefore

$$
\sup _{v \in \mathscr{F}} \int_{c}^{d} f(s, v(s)) d s \leq \int_{c}^{d} \sup _{y \in \mathbb{R}} f(s, y) d s .
$$

From (2), it follows that for each $\varepsilon>0$ and for each $s \in[c, d]$, there exists a $j \in \mathbb{N}$ such that $E(s)-\varepsilon<f\left(s, y_{j}\right)$. For each $j \in \mathbb{N}$, define sets $M_{j}$ and $P_{j}$ by

$$
M_{j}=\left\{s: E(s)-\varepsilon<f\left(s, y_{j}\right) \text { and } E(s)-\varepsilon \geq f\left(s, y_{i}\right) \text { for all } i<j\right\},
$$

and

$$
P_{j}=\left\{s: E(s)-\varepsilon<f\left(s, y_{j}\right)\right\} \text {. }
$$


Fix any $j \in \mathbb{N}$. Since $E(s)-f\left(s, y_{j}\right)$ is Lebesgue measurable, we see that $P_{j}$ is a Lebesgue measurable set. Hence, $M_{j}=P_{j} \backslash \bigcup_{i<j} P_{i}$ is also a Lebesgue measurable set. Now define $\bar{v}:[c, d] \rightarrow \mathbb{R}$ by

$$
\bar{v}(s)=y_{j} \quad \text { for } s \in M_{j}, j \in \mathbb{N} .
$$

Since $M_{j}$ is measurable for each $j \in \mathbb{N}$, we have $\bar{v} \in \mathscr{F}$. Then,

$$
\begin{aligned}
\int_{c}^{d}[E(s)-\varepsilon] d s & =\sum_{j=1}^{\infty} \int_{M_{j}}[E(s)-\varepsilon] d s<\sum_{j=1}^{\infty} \int_{M_{j}} f\left(s, y_{j}\right) d s \\
& =\int_{c}^{d} f(s, \bar{v}(s)) d s \leq \sup _{v \in \mathscr{F}} \int_{c}^{d} f(s, v(s)) d s .
\end{aligned}
$$

Now let $\varepsilon \downarrow 0$. We thus have

$$
\int_{c}^{d} \sup _{y \in \mathbf{R}} f(s, y) d s \leq \sup _{v \in \mathscr{F}} \int_{c}^{d} f(s, v(s)) d s .
$$

This inequality, along with (3), gives the desired result.

Lemma 2. Let any $T>0$ and $x_{0} \in \mathbb{R}$ be given. Let $\left\{f_{k}\right\}_{k \in \mathbb{N}}$, $f_{\infty}$ be functions which satisfy properties (P1), (P2), (P3) and (P4). Assume also that

(i) $\left\{f_{k}\right\}_{k \in \mathbb{N}}, f_{\infty}$ are uniformly bounded, and

(ii) for each $c, d \in[0, T]$ with $c \leq d$,

$$
\limsup _{k \rightarrow \infty} \int_{c}^{d} \sup _{y \in \mathbb{R}}\left[f_{k}(s, y)-f_{\infty}(s, y)\right] d s \leq 0 .
$$

For $k=1,2, \ldots, \infty$, let $m_{k}$ denote the greatest solution to the initial value problem

$$
m_{k}^{\prime}(t)=f_{k}\left(t, m_{k}(t)\right), \quad \text { for } t \in[0, T], \quad m_{k}(0)=x_{0} .
$$

Then, for all $n \in \mathbb{N}$ and all $\varepsilon>0$,

$$
\limsup _{k \rightarrow \infty}\left\{\sup _{t \in[0, T]}\left[m_{k}(t)-x_{n, \varepsilon}(t)\right]\right\} \leq \varepsilon,
$$

where $x_{n, \varepsilon}$ is defined as in the proof of Theorem 1, in terms of $f_{\infty}$ and the uniform bound of $\left\{f_{k}\right\}_{k \in \mathbb{N}}, f_{\infty}$.

Proof. Let any $n \in \mathbb{N}$ and any $\varepsilon>0$ be given. These will remain fixed throughout this proof. Let $M$ denote the uniform bound of $\left\{f_{k}\right\}_{k \in \mathbb{N}}, f_{\infty}$.

Claim. For each $\hat{\varepsilon}>0$, there exists a $K \in \mathbb{N}$ such that

$$
\int_{c}^{d} f_{k}(s, v(s)) d s \leq \int_{c}^{d} f_{\infty}(s, v(s)) d s+\hat{\varepsilon}
$$

for all $k \geq K$, for all $c, d$ with $0 \leq c \leq d \leq T$ and for all Lebesgue measurable functions $v:[c, d] \rightarrow \mathbb{R}$.

Proof of Claim. Fix $\hat{\varepsilon}>0$. From hypothesis (ii) and Lemma 1, it follows that for all $c, d$ with $0 \leq c \leq d \leq T$ there exists a $K=K(c, d) \in \mathbb{N}$ such that

$$
\int_{c}^{d}\left[f_{k}(s, v(s))-f_{\infty}(s, v(s))\right] d s \leq \hat{\varepsilon}
$$


for all $k \geq K$ and for all $v \in \mathscr{F} \equiv\{v:[c, d] \rightarrow \mathbb{R} \mid v$ is Lebesgue measurable $\}$. What needs to be shown is that $K$ can be chosen independently of $c$ and $d$. Choose $J$ to be an integer such that

$$
J \geq 8 M T / \hat{\varepsilon} .
$$

Let $t_{i}=i T / J$, for $i=0,1,2, \ldots, J$. For each $i, j \in\{0,1, \ldots, J\}$ with $i \leq j$, there exists some $K_{i j}$ such that

$$
\int_{t_{i}}^{t_{j}} f_{k}(s, v(s)) d s \leq \int_{t_{i}}^{t_{j}} f_{\infty}(s, v(s)) d s+\frac{\hat{\varepsilon}}{2}
$$

for all $k \geq K_{i j}$ and for all $v \in \mathscr{F}$. Hence, letting $K=\max _{i, j} K_{i j}$, we have

$$
\int_{t_{i}}^{t_{j}} f_{k}(s, v(s)) d s \leq \int_{t_{i}}^{t_{j}} f_{\infty}(s, v(s)) d s+\frac{\hat{\varepsilon}}{2}
$$

for all $i, j \in\{0,1, \ldots, J\}$ with $i \leq j$, for all $k \geq K$ and for all $v \in \mathscr{F}$. Now let any $c, d$ with $0 \leq c \leq d \leq T$ be given. Choose $i, j$ such that $t_{i-1} \leq c \leq t_{i}, t_{j-1} \leq d \leq t_{j}$ and $i \leq j$. Then,

$$
\begin{aligned}
\int_{c}^{d}[ & \left.f_{k}(s, v(s))-f_{\infty}(s, v(s))\right] d s \\
= & \int_{c}^{t_{i}}\left[f_{k}(s, v(s))-f_{\infty}(s, v(s))\right] d s \\
& \quad+\int_{t_{i}}^{t_{j}}\left[f_{k}(s, v(s))-f_{\infty}(s, v(s))\right] d s \\
& \quad-\int_{d}^{t_{j}}\left[f_{k}(s, v(s))-f_{\infty}(s, v(s))\right] d s
\end{aligned}
$$

(Note the use of absolute values in two of the following integrands.)

$$
\begin{aligned}
\leq & \int_{c}^{t_{i}}\left|f_{k}(s, v(s))-f_{\infty}(s, v(s))\right| d s \\
& +\int_{t_{i}}^{t_{j}}\left[f_{k}(s, v(s))-f_{\infty}(s, v(s))\right] d s \\
& +\int_{d}^{t_{j}}\left|f_{k}(s, v(s))-f_{\infty}(s, v(s))\right| d s \\
& \stackrel{(6)}{\leq} 2 M T / J+\hat{\varepsilon} / 2+2 M T / J \stackrel{(5)}{\leq} \hat{\varepsilon} .
\end{aligned}
$$

Now that the claim is established, we choose $\hat{\varepsilon}=\varepsilon / l$ (where $l$ is the number of subintervals in the partition of $[0, T]$ for $x_{n, \varepsilon}$ ) and then choose $k$ so that (4) holds.

Claim. For each $j=0,1, \ldots, l-1$ and for $t \in\left[\sigma_{j}^{(n, \varepsilon)}, \sigma_{j+1}^{(n, \varepsilon)}\right]$,

$$
m_{k}(t) \leq x_{n, \varepsilon}(t)+(j+1) \hat{\varepsilon} \text {. }
$$

Proof of Claim. We shall handle this claim inductively. First, consider $t \in$ $\left[\sigma_{0}^{(n, \varepsilon)}, \sigma_{1}^{(n, \varepsilon)}\right]$. Recall that $\sigma_{0}^{(n, \varepsilon)}=0$ and $x_{n, \varepsilon}(0)=x_{0}=m_{k}(0)$. We then 
have

$$
\begin{aligned}
& \left|m_{k}(t)-x_{n, \varepsilon}\left(\sigma_{0}^{(n, \varepsilon)}\right)\right|=\left|m_{k}(t)-m_{k}\left(\sigma_{0}^{(n, \varepsilon)}\right)\right| \\
& =\left|\int_{\sigma_{0}^{(n, \varepsilon)}}^{t} f_{k}\left(s, m_{k}(s)\right) d s\right| \leq M\left(t-\sigma_{0}^{(n, \varepsilon)}\right) \leq 2 M\left(t-\sigma_{0}^{(n, \varepsilon)}\right)+\varepsilon .
\end{aligned}
$$

Thus,

$$
\begin{aligned}
m_{k}(t) & =m_{k}\left(\sigma_{0}^{(n, \varepsilon)}\right)+\int_{\sigma_{0}^{(n, \varepsilon)}}^{t} f_{k}\left(s, m_{k}(s)\right) d s \\
& \stackrel{(4)}{\leq} x_{n, \varepsilon}\left(\sigma_{0}^{(n, \varepsilon)}\right)+\int_{\sigma_{0}^{(n, \varepsilon)}}^{t} f_{\infty}\left(s, m_{k}(s)\right) d s+\hat{\varepsilon} \\
& \stackrel{(7)}{\leq} x_{n, \varepsilon}\left(\sigma_{0}^{(n, \varepsilon)}\right)+\int_{\sigma_{0}^{(n, \varepsilon)}}^{t} \sup \left\{f_{\infty}(s, x):\left|x-x_{n, \varepsilon}\left(\sigma_{0}^{(n, \varepsilon)}\right)\right|\right. \\
& \left.\leq 2 M\left(s-\sigma_{0}^{(n, \varepsilon)}\right)+\varepsilon\right\} d s+\hat{\varepsilon} \\
& =x_{n, \varepsilon}(t)+\hat{\varepsilon} .
\end{aligned}
$$

Now let $j \in \mathbb{N}$ with $0<j<l$ be given and assume $m_{k}\left(\sigma_{j}^{(n, \varepsilon)}\right) \leq x_{n, \varepsilon}\left(\sigma_{j}^{(n, \varepsilon)}\right)$ $+j \hat{\varepsilon}$.

Case One. $m_{k}\left(\sigma_{j}^{(n, \varepsilon)}\right) \leq x_{n, \varepsilon}\left(\sigma_{j}^{(n, \varepsilon)}\right)$.

Subcase (i). $m_{k}(t)<x_{n, \varepsilon}(t)$ for all $t \in\left[\sigma_{j}^{(n, \varepsilon)}, \sigma_{j+1}^{(n, \varepsilon)}\right]$.

Obviously, $m_{k}(t) \leq x_{n, \varepsilon}(t)+(j+1) \hat{\varepsilon}$ for all $t \in\left[\sigma_{j}^{(n, \varepsilon)}, \sigma_{j+1}^{(n, \varepsilon)}\right]$.

Subcase (ii). There exists some $a \in\left[\sigma_{j}^{(n, \varepsilon)}, \sigma_{j+1}^{(n, \varepsilon)}\right]$ such that

$$
m_{k}(a)=x_{n, \varepsilon}(a) \text { and } m_{k}(s)<x_{n, \varepsilon}(s) \text { for all } s \in\left[\sigma_{j}^{(n, \varepsilon)}, a\right)
$$

Fix any $t \in\left[a, \sigma_{j+1}^{(n, \varepsilon)}\right]$. Then, for $s \in[a, t]$,

(8)

$$
\begin{aligned}
\mid m_{k}(s) & -x_{n, \varepsilon}\left(\sigma_{j}^{(n, \varepsilon)}\right) \mid \\
\leq & \left|m_{k}(s)-m_{k}(a)\right|+\left|x_{n, \varepsilon}(a)-x_{n, \varepsilon}\left(\sigma_{j}^{(n, \varepsilon)}\right)\right| \\
= & \left|\int_{a}^{s} f_{k}\left(r, m_{k}(r)\right) d r\right| \\
& +\left|\int_{\sigma_{j}^{(n, \varepsilon)}}^{a} \sup \left\{f_{\infty}(r, x):\left|x-x_{n, \varepsilon}\left(\sigma_{j}^{(n, \varepsilon)}\right)\right| \leq 2 M\left(r-\sigma_{j}^{(n, \varepsilon)}\right)+\varepsilon\right\} d r\right| \\
\leq & M(s-a)+M\left(a-\sigma_{j}^{(n, \varepsilon)}\right) \leq 2 M\left(s-\sigma_{j}^{(n, \varepsilon)}\right)+\varepsilon .
\end{aligned}
$$


Therefore,

$$
\begin{aligned}
m_{k}(t) & =m_{k}(a)+\int_{a}^{t} f_{k}\left(s, m_{k}(s)\right) d s \\
& \stackrel{(4)}{\leq} x_{n, \varepsilon}(a)+\int_{a}^{t} f_{\infty}\left(s, m_{k}(s)\right) d s+\hat{\varepsilon} \\
& \stackrel{(8)}{\leq} x_{n, \varepsilon}(a)+\int_{a}^{t} \sup \left\{f_{\infty}(s, x):\left|x-x_{n, \varepsilon}\left(\sigma_{j}^{(n, \varepsilon)}\right)\right|\right. \\
& \left.\leq 2 M\left(s-\sigma_{j}^{(n, \varepsilon)}\right)+\varepsilon\right\} d s+\hat{\varepsilon} \\
& =x_{n, \varepsilon}(t)+\hat{\varepsilon} \leq x_{n, \varepsilon}(t)+(j+1) \hat{\varepsilon} .
\end{aligned}
$$

Case Two.

$$
x_{n, \varepsilon}\left(\sigma_{j}^{(n, \varepsilon)}\right)<m_{k}\left(\sigma_{j}^{(n, \varepsilon)}\right) \leq x_{n, \varepsilon}\left(\sigma_{j}^{(n, \varepsilon)}\right)+j \hat{\varepsilon} .
$$

Note first that we have

$$
0<m_{k}\left(\sigma_{j}^{(n, \varepsilon)}\right)-x_{n, \varepsilon}\left(\sigma_{j}^{(n, \varepsilon)}\right) \leq j \hat{\varepsilon}
$$

and hence

$$
\left|m_{k}\left(\sigma_{j}^{(n, \varepsilon)}\right)-x_{n, \varepsilon}\left(\sigma_{j}^{(n, \varepsilon)}\right)\right| \leq j \hat{\varepsilon} .
$$

Fix $t \in\left[\sigma_{j}^{(n, \varepsilon)}, \sigma_{j+1}^{(n, \varepsilon)}\right]$. Then, for each $s \in\left[\sigma_{j}^{(n, \varepsilon)}, t\right]$, we have

$$
\begin{gathered}
\left|m_{k}(s)-x_{n, \varepsilon}\left(\sigma_{j}^{(n, \varepsilon)}\right)\right| \leq\left|m_{k}(s)-m_{k}\left(\sigma_{j}^{(n, \varepsilon)}\right)\right|+\left|m_{k}\left(\sigma_{j}^{(n, \varepsilon)}\right)-x_{n, \varepsilon}\left(\sigma_{j}^{(n, \varepsilon)}\right)\right| \\
\quad \stackrel{(10)}{\leq} M\left(s-\sigma_{j}^{(n, \varepsilon)}\right)+j \hat{\varepsilon}<2 M\left(s-\sigma_{j}^{(n, \varepsilon)}\right)+l \hat{\varepsilon}=2 M\left(s-\sigma_{j}^{(n, \varepsilon)}\right)+\varepsilon .
\end{gathered}
$$

Thus,

$$
\begin{aligned}
m_{k}(t) & =m_{k}\left(\sigma_{j}^{(n, \varepsilon)}\right)+\int_{\sigma_{j}^{(n, \varepsilon)}}^{t} f_{k}\left(s, m_{k}(s)\right) d s \\
\stackrel{(4),(9)}{\leq} & x_{n, \varepsilon}\left(\sigma_{j}^{(n, \varepsilon)}\right)+j \hat{\varepsilon}+\int_{\sigma_{j}^{(n, \varepsilon)}}^{t} f_{\infty}\left(s, m_{k}(s)\right) d s+\hat{\varepsilon} \\
& \leq x_{n, \varepsilon}\left(\sigma_{j}^{(n, \varepsilon)}\right)+\int_{\sigma_{j}^{(n, \varepsilon)}}^{t} \sup \left\{f_{\infty}(s, x):\left|x-x_{n, \varepsilon}\left(\sigma_{j}^{(n, \varepsilon)}\right)\right|\right. \\
& =x_{n, \varepsilon}(t)+(j+1) \hat{\varepsilon} .
\end{aligned}
$$

We have now established the claim. From this it follows that

$$
m_{k}(t) \leq x_{n, \varepsilon}(t)+l \hat{\varepsilon}=x_{n, \varepsilon}(t)+\varepsilon \text { for all } t \in[0, T]
$$

(recall that $T=\sigma_{l}^{(n, \varepsilon)}$ ).

We have shown for all $\varepsilon>0$ and all $n \in \mathbb{N}$, there exists a $K \in \mathbb{N}$ such that for all $k \geq K$, we have

$$
m_{k}(t) \leq x_{n, \varepsilon}(t)+\varepsilon \text { for all } t \in[0, T]
$$

That is,

as desired.

$$
\limsup _{k \rightarrow \infty}\left\{\sup _{t \in[0, T]}\left[m_{k}(t)-x_{n, \varepsilon}(t)\right]\right\} \leq \varepsilon
$$


Theorem 2. Let any $T>0$ and $x_{0} \in \mathbb{R}$ be given. Let $\left\{f_{k}\right\}_{k \in \mathbb{N}}, f_{\infty}$ be functions which satisfy properties (P1), (P2), (P3) and (P4).

Assume also that $\left\{f_{k}\right\}_{k \in \mathbb{N}}, f_{\infty}$ are uniformly bounded. For $k=1,2, \ldots, \infty$, let $m_{k}$ denote the greatest solution to the initial value problem

$$
\begin{aligned}
& m_{k}^{\prime}(t)=f_{k}\left(t, m_{k}(t)\right) \quad \text { for } t \in[0, T], \\
& m_{k}(0)=x_{0} .
\end{aligned}
$$

Also assume that for each $c, d \in[0, T]$ with $c \leq d$,

$$
\underset{k \rightarrow \infty}{\limsup } \int_{c}^{d} \sup _{y \in \mathbb{R}}\left[f_{k}(s, y)-f_{\infty}(s, y)\right] d s \leq 0 .
$$

Then

$$
\limsup _{k \rightarrow \infty}\left\{\sup _{t \in[0, T]}\left[m_{k}(t)-m_{\infty}(t)\right]\right\} \leq 0 .
$$

Proof. Consider $\mathscr{U} \equiv\left\{u_{\varepsilon}: \varepsilon \geq 0\right\}$, where $u_{\varepsilon}$ is defined as in the proof of Theorem 1, using $f_{\infty}$ and $M$, the uniform bound of $\left\{f_{k}\right\}_{k \in \mathbb{N}}, f_{\infty}$. We note that for $\varepsilon \geq 0, n \in \mathbb{N}$ and $t_{1}, t_{2} \in[0, T]$,

$$
\left|x_{n, \varepsilon}\left(t_{2}\right)-x_{n, \varepsilon}\left(t_{1}\right)\right| \leq\left|\int_{t_{1}}^{t_{2}} a_{n, \varepsilon}(s) d s\right| \leq M\left|t_{2}-t_{1}\right|,
$$

i.e., $\left\{x_{n, \varepsilon}\right\}$ is uniformly Lipschitz. Hence, $\left\{u_{\varepsilon}\right\}$ is also, and so $\mathscr{U}$ is equicontinuous. Also, since $u_{\varepsilon}(0)=x_{0}$ for all $\varepsilon \geq 0$, it follows from the uniform Lipschitz property that $\mathscr{U}$ is uniformly bounded. Thus, we have a uniformly bounded and equicontinuous family of continuous functions defined on the compact set $[0, T]$. Hence, by the Ascoli-Arzela lemma, there is some continuous function $v$ and some subsequence $\left\{\varepsilon_{i}\right\}_{i=1}^{\infty}$ such that $\varepsilon_{i} \downarrow 0$ and $u_{\varepsilon_{i}} \rightarrow v$ uniformly on $[0, T]$ as $i \rightarrow \infty$.

Fix any $t \in[0, T)$. Choose any $i, n \in \mathbb{N}$. There exists some $j \in \mathbb{N}$ such that $t \in\left[\sigma_{j}^{\left(n, \varepsilon_{i}\right)}, \sigma_{j+1}^{\left(n, \varepsilon_{i}\right)}\right)$. We have

$$
\left|x_{n, \varepsilon_{i}}(t)-x_{n, \varepsilon_{i}}\left(\sigma_{j}^{\left(n, \varepsilon_{i}\right)}\right)\right|=\left|\int_{\sigma_{j}^{\left(n, \varepsilon_{i}\right)}}^{t} a_{n, \varepsilon_{i}}(s) d s\right| \leq M\left(t-\sigma_{j}^{\left(n, \varepsilon_{i}\right)}\right) .
$$

Since

$$
a_{n, \varepsilon_{i}}(t)=\sup \left\{f_{\infty}(t, x):\left|x-x_{n, \varepsilon_{i}}\left(\sigma_{j}^{\left(n, \varepsilon_{i}\right)}\right)\right| \leq 2 M\left(t-\sigma_{j}^{\left(n, \varepsilon_{i}\right)}\right)+\varepsilon_{i}\right\},
$$

there exists some $\widehat{x_{n, \varepsilon_{i}}} \in \mathbb{R}$ satisfying

$$
\left|\widehat{x_{n, \varepsilon_{i}}}-x_{n, \varepsilon_{i}}\left(\sigma_{j}^{\left(n, \varepsilon_{i}\right)}\right)\right| \leq 2 M\left(t-\sigma_{j}^{\left(n, \varepsilon_{i}\right)}\right)+\varepsilon_{i}
$$

and

$$
a_{n, \varepsilon_{i}}(t)-2^{-n} \leq f_{\infty}\left(t, \widehat{x_{n, \varepsilon_{i}}}\right) \leq a_{n, \varepsilon_{i}}(t) .
$$

Hence,

$$
\limsup _{n \rightarrow \infty} a_{n, \varepsilon_{i}}(t)=\limsup _{n \rightarrow \infty} f_{\infty}\left(t, \widehat{x_{n, \varepsilon_{i}}}\right) .
$$

Note that for each $i, n \in \mathbb{N}$, we have

$$
\left|\widehat{x_{n, \varepsilon_{i}}}-x_{n, \varepsilon_{i}}(t)\right| \stackrel{(11),(12)}{\leq} 2 M\left(t-\sigma_{j}^{\left(n, \varepsilon_{i}\right)}\right)+\varepsilon_{i}+M\left(t-\sigma_{j}^{\left(n, \varepsilon_{i}\right)}\right) \leq \frac{3 M T}{2^{n}}+\varepsilon_{i}
$$


Fix any $i \in \mathbb{N}$. Let $B_{i} \equiv \lim \sup _{n \rightarrow \infty} f_{\infty}\left(t, \widehat{x_{n, \varepsilon_{i}}}\right)$. Choose $\left\{n_{k}\right\}$, depending on $i$, such that $f_{\infty}\left(t, \widehat{x_{n_{k}}, \varepsilon_{i}}\right) \rightarrow B_{i}$ as $k \rightarrow \infty$. From (14), we know that $\left\{\widehat{x_{n_{k}, \varepsilon_{i}}}\right\}_{k=1}^{\infty}$ is bounded. Hence, replacing $\left\{n_{k}\right\}$ with a suitable subsequence, we may assume $\left\{\widehat{x_{n_{k}, \varepsilon_{i}}}\right\}_{k=1}^{\infty}$ approaches some limit $\lambda_{i}$ as $k \rightarrow \infty$. We then have

$$
\boldsymbol{B}_{i}=\lim _{k \rightarrow \infty} f_{\infty}\left(t, \widehat{x_{n_{k}}, \varepsilon_{i}}\right)=\underset{k \rightarrow \infty}{\limsup } f_{\infty}\left(t, \widehat{x_{n_{k}}, \varepsilon_{i}}\right) \leq f_{\infty}\left(t, \lambda_{i}\right)
$$

from our hypotheses on $f_{\infty}$. Note also that (14) implies $\left|\lambda_{i}-u_{\varepsilon_{i}}(t)\right| \leq \varepsilon_{i}$, hence $\lambda_{i} \rightarrow v(t)$ as $i \rightarrow \infty$. We thus have

$$
\limsup _{i \rightarrow \infty} B_{i} \stackrel{(15)}{\leq} \limsup _{i \rightarrow \infty} f_{\infty}\left(t, \lambda_{i}\right) \leq f_{\infty}(t, v(t)) .
$$

Therefore, for each $t \in[0, T)$,

(17) $\quad \limsup \limsup _{n \rightarrow \infty} a_{n, \varepsilon_{i}}(t) \stackrel{(13)}{=} \limsup _{i \rightarrow \infty} \limsup _{n \rightarrow \infty} f_{\infty}\left(t, \widehat{x_{n, \varepsilon_{i}}}\right) \stackrel{(16)}{\leq} f_{\infty}(t, v(t))$.

Now fix any $t_{1}, t_{2} \in[0, T]$ with $t_{1} \leq t_{2}$. Recalling that

$$
x_{n, \varepsilon_{i}}\left(t_{2}\right)=x_{n, \varepsilon_{i}}\left(t_{1}\right)+\int_{t_{1}}^{t_{2}} a_{n, \varepsilon_{i}}(s) d s,
$$

we have, after two applications of Fatou's lemma and the use of (17),

$$
v\left(t_{2}\right) \leq v\left(t_{1}\right)+\int_{t_{1}}^{t_{2}} f_{\infty}(s, v(s)) d s .
$$

Note also that for any $i, n \in \mathbb{N}$ and $t_{1}, t_{2} \in[0, T]$ with $t_{1} \leq t_{2}$,

$$
\left|x_{n, \varepsilon_{i}}\left(t_{2}\right)-x_{n, \varepsilon_{i}}\left(t_{1}\right)\right|=\left|\int_{t_{1}}^{t_{2}} a_{n, \varepsilon_{i}}(s) d s\right| \leq M\left(t_{2}-t_{1}\right) \text {. }
$$

Hence,

$$
\left|v\left(t_{2}\right)-v\left(t_{1}\right)\right| \leq M\left(t_{2}-t_{1}\right)
$$

Claim. $v(t) \leq m_{\infty}(t)$ for all $t \in[0, T]$.

Proof of Claim. Suppose not. Then, there exists some $\bar{t} \in(0, T)$ such that $v(\bar{t})>m_{\infty}(\bar{t})$. From the proof of Theorem 1, we have $x_{n, 0}(\bar{t}) \downarrow m_{\infty}(\bar{t})$. Hence, choosing $N$ large enough, we have $v(\bar{t})>x_{N, 0}(\bar{t})$. Since $v(0)=x_{0}=x_{N, 0}(0)$, there exists a subinterval $\left[\sigma_{j}^{(N, 0)}, \sigma_{j+1}^{(N, 0)}\right]$ and $t_{1}, t_{2} \in\left[\sigma_{j}^{(N, 0)}, \sigma_{j+1}^{(N, 0)}\right]$ with $t_{1}<t_{2}$ such that $v\left(t_{1}\right)=x_{N, 0}\left(t_{1}\right)$ and

$$
v(t)>x_{N, 0}(t) \text { for } t \in\left(t_{1}, t_{2}\right] .
$$

Note that for $t \in\left(t_{1}, t_{2}\right]$, we have

$$
\begin{aligned}
\left|v(t)-x_{N, 0}\left(\sigma_{j}^{(N, 0)}\right)\right| & \leq\left|v(t)-v\left(t_{1}\right)\right|+\left|x_{N, 0}\left(t_{1}\right)-x_{N, 0}\left(\sigma_{j}^{(N, 0)}\right)\right| \\
& \stackrel{(19)}{\leq} M\left(t-t_{1}\right)+\int_{\sigma_{j}^{(N, 0)}}^{t_{1}}\left|a_{N, 0}(s)\right| d s \\
& \leq M\left(t-t_{1}\right)+M\left(t_{1}-\sigma_{j}^{(N, 0)}\right) \\
& \leq 2 M\left(t-\sigma_{j}^{(N, 0)}\right) .
\end{aligned}
$$


Hence,

$$
\begin{aligned}
v\left(t_{2}\right) & \stackrel{(18)}{\leq} v\left(t_{1}\right)+\int_{t_{1}}^{t_{2}} f_{\infty}(t, v(t)) d t \\
& \leq x_{N, 0}\left(t_{1}\right)+\int_{t_{1}}^{t_{2}} \sup \left\{f_{\infty}(t, x):\left|x-x_{N, 0}\left(\sigma_{j}^{(N, 0)}\right)\right| \leq 2 M\left(t-\sigma_{j}^{(N, 0)}\right)\right\} d t \\
& =x_{N, 0}\left(t_{2}\right)
\end{aligned}
$$

contradicting (20). This proves the claim.

Now let any $\gamma>0$ be given. We wish to show there exists some $K=K(\gamma)$ such that for all $k \geq K$, we have

$$
m_{k}(t) \leq m_{\infty}(t)+\gamma \quad \text { for all } t \in[0, T] .
$$

Since $u_{\varepsilon_{i}}(t) \rightarrow v(t)$ uniformly on $[0, T]$, there exists some $\bar{\varepsilon}=\bar{\varepsilon}(\gamma)$ such that

$$
u_{\bar{\varepsilon}}(t)+2 \bar{\varepsilon} \leq v(t)+\gamma \text { for all } t \in[0, T] .
$$

In the proof of Theorem 1 , we showed that for each $\varepsilon \geq 0, x_{n, \varepsilon}(t) \rightarrow u_{\varepsilon}(t)$ uniformly on $[0, T]$. Hence, there exists some $N=N(\gamma)$ such that

$$
x_{N, \bar{\varepsilon}}(t)+\bar{\varepsilon} \leq u_{\bar{\varepsilon}}(t)+2 \bar{\varepsilon} \quad \text { for all } t \in[0, T] .
$$

From Lemma 2, we know there exists some $K=K(\gamma)$ such that for all $k \geq K$,

$$
m_{k}(t) \leq x_{N, \bar{\varepsilon}}(t)+\bar{\varepsilon} \quad \text { for all } t \in[0, T] .
$$

We then have, for all $k \geq K$, the following inequalities holding for all $t \in$ $[0, T]$ :

$$
m_{k}(t) \leq x_{N, \bar{\varepsilon}}(t)+\bar{\varepsilon} \leq u_{\bar{\varepsilon}}(t)+2 \bar{\varepsilon} \leq v(t)+\gamma \leq m_{\infty}(t)+\gamma .
$$

This establishes (21), and so the theorem is proven.

To help clarify Theorem 2 , we give the following interpretation. We may be interested in the solutions $u$ (greatest or not) of $u^{\prime}(t)=f_{k}(t, u(t))$, but these may be difficult to determine. If we can find an $f_{\infty}$ which satisfies the theorem's hypotheses for which $m_{\infty}$ is easy to determine, the theorem yields an approximate upper bound on $u$ : roughly, as $k$ gets large, $u$ cannot be much larger than $m_{\infty}$.

\section{EXAMPLES AND DISCUSSION}

We now discuss the result proven in the previous section and present examples to illustrate its principal features.

For the reader who is familiar with the Filippov notion of solution (see [4]), this paper may at first glance seem to be a special case of that theory. However, since a Filippov solution is not necessarily a Carathéodory solution, Theorem 1 is needed. Theorem 2 is also needed. Although there are continuous dependence theorems for Filippov solutions, none appear to include the convergence criterion used in Theorem 2 as a special case. For example, consider the convergence criterion used in [4]:

$$
\lim _{k \rightarrow \infty} \int_{0}^{T} \Psi_{k}(s) d s=0,
$$


where $\left|f_{k}(s, y)-f_{\infty}(s, y)\right| \leq \Psi_{k}(s)$ for almost all $(s, y)$. This type of convergence is more restrictive than that used in Theorem 2: take, for example, $f_{k}(s, y)=\sin (k s)$ and $f_{\infty}(s, y) \equiv 0$.

Next, the lim sup's and inequalities in Theorem 2 may appear unnatural and somewhat removed from the theory discussed in the Introduction. However, consider the following. For $k=1,2, \ldots$, let

$$
f_{k}(t, x)= \begin{cases}-1, & \text { for } x<0 \\ -\frac{1}{k}, & \text { for } x \geq 0\end{cases}
$$

Also, let

$$
f_{\infty}(t, x)= \begin{cases}-1, & \text { for } x<0, \\ 0, & \text { for } x \geq 0 .\end{cases}
$$

We note that these functions, for $k=1,2, \ldots, \infty$, satisfy properties (P1), (P2), (P3) and (P4) (with any $T>0$ ). We also note that the $f_{k}$ 's converge to $f_{\infty}$ in most any sense one may consider, for example, pointwise. It is easily shown that the greatest solution to $\left(\operatorname{IVP}^{\prime}\right)$ with $x_{0}=0$ is given by $m_{k}(t)=-t$ for $k=1,2, \ldots$ and $m_{\infty}(t) \equiv 0$. Thus, $m_{k}$ does not converge pointwise to $m_{\infty}$. But, we do have

$$
\limsup _{k \rightarrow \infty} m_{k}(t)=-t \leq 0=m_{\infty}(t)
$$

for $t \geq 0$. Thus, this type of conclusion is the best we can obtain for $f$ 's which satisfy properties (P1), (P2), (P3) and (P4). Theorem 2 proves that we can also assume a correspondingly weak convergence on the $f$ 's. Schechter referred to such a phenomenon as "one-sided continuous dependence" in [10]. We also note that the $m_{k}$ 's $d o$ converge to a solution of $x^{\prime}=f_{\infty}(t, x), x(0)=0$, namely $u_{\infty}(t)=-t$. Thus, it is possible that in this context (and others) that it is unnatural to restrict ourselves to greatest Carathéodory solutions. That is, it may be that "nicer" continuous dependence results may be obtained by considering, for example, how the sets of solutions relate.

We next note that the convergence on the $f$ 's in Theorem 2 is not necessary for the convergence on the $m$ 's required there, evidence of which is provided by the following example. For $k=1,2, \ldots$, let $f_{k}$ be given by

$$
f_{k}(t, x)= \begin{cases}0, & \text { for } x<0, \\ 1-k x, & \text { for } x \in\left[0, \frac{1}{k}\right], \\ 0, & \text { for } x \geq \frac{1}{k},\end{cases}
$$

and let $f_{\infty}(t, x) \equiv 0$. We note that for $k=1,2, \ldots, \infty, f_{k}$ satisfies properties (P1), (P2), (P3) and (P4) (with any $T>0$ ). It can be shown that for any $T>0$ and $x_{0} \in \mathbb{R}$ we have

$$
\underset{k \rightarrow \infty}{\limsup }\left\{\sup _{t \in[0, T]}\left[m_{k}(t)-m_{\infty}(t)\right]\right\} \leq 0,
$$

that is, the conclusion of Theorem 2 holds. (The details are rather tedious.) 
However, we have

$$
\begin{aligned}
\underset{k \rightarrow \infty}{\limsup } \int_{0}^{T} \sup _{y \in \mathbb{R}}\left[f_{k}(s, y)-f_{\infty}(s, y)\right] d s \\
=\limsup _{k \rightarrow \infty} \int_{0}^{T}\left[f_{k}(s, 0)-f_{\infty}(s, 0)\right] d s \\
=\underset{k \rightarrow \infty}{\limsup } \int_{0}^{T} 1 d s=T>0,
\end{aligned}
$$

that is, the convergence of the $f$ 's used in Theorem 2 does not hold. In fact, we note that this set of $f$ 's would not satisfy most any notion of convergence on the $f$ 's that might guarantee the conclusion of Theorem 2. That is, it is not at all clear just what type of convergence on the $f$ 's, if any, might be necessary and sufficient for (22).

Finally, we note that the "global' $\sup _{y \in \mathbb{R}}$ device used in Theorem 2 for the convergence criterion on the $f$ 's may appear unnatural, since the convergence criterion is "local" in the time variable. For example, one may conjecture that the following is sufficient for the conclusion of Theorem 2:

For each $c, d \in[0, T]$ with $c \leq d$ and for each $y \in \mathbb{R}$,

$$
\limsup _{k \rightarrow \infty} \int_{c}^{d}\left[f_{k}(s, y)-f_{\infty}(s, y)\right] d s \leq 0 .
$$

(See [10] for a use of (23).) However, consider the following example. For $k=1,2, \ldots$, let $f_{k}:(0,1) \times \mathbb{R} \rightarrow \mathbb{R}$ be given by

$$
f_{k}(t, x)=\left\{\begin{array}{l}
\left(\frac{-k-1}{t}\right) x+\frac{(k+1)(k+2)}{k} \\
\quad \text { for }\left(\frac{k+1}{k}\right) t \leq x \leq\left(\frac{k+2}{k}\right) t, \\
0, \quad \text { elsewhere }
\end{array}\right.
$$

and let $f_{\infty}:(0,1) \times \mathbb{R} \rightarrow \mathbb{R}$ be given by $f_{\infty}(t, x) \equiv 0$. (For fixed $t, f_{k}$ simply defines a line segment between the point $x=\left(\frac{k+1}{k}\right) t, f_{k}=\frac{k+1}{k}$ and the point $x=\left(\frac{k+2}{k}\right) t, f_{k}=0$.) Note that, for $k=1,2, \ldots, \infty, f_{k}$ satisfies properties (P1), (P2), (P3) and (P4), and that $\left\{f_{k}\right\}_{k \in \mathbb{N}}, f_{\infty}$ are uniformly bounded. Take $\left(\mathrm{IVP}^{\prime}\right)$ with $T=1$ and $x_{0}=0$. It can be shown that for $k=1,2, \ldots, m_{k}(t)=\left(\frac{k+1}{k}\right) t$ and $m_{\infty}(t) \equiv 0$. It can also be shown, albeit tediously, that $(23)$ is satisfied. However, we have

$$
\begin{aligned}
\underset{k \rightarrow \infty}{\limsup } & \left\{\sup _{t \in[0,1]}\left[m_{k}(t)-m_{\infty}(t)\right]\right\} \\
= & \limsup _{k \rightarrow \infty}\left\{\sup _{t \in[0,1]}\left[\left(\frac{k+1}{k}\right) t\right]\right\} \\
= & \limsup _{k \rightarrow \infty} \frac{k+1}{k}=1>0,
\end{aligned}
$$


that is, the conclusion to Theorem 2 does not hold. Therefore, this example demonstrates that $(23)$ is not sufficient.

\section{ACKNOWLEDGMENTS}

The author wishes to thank the referee for an extremely thorough report, which included a proof of the characterization of the $u_{\varepsilon}$ 's mentioned after the proof of Theorem 1 and also included many suggestions which greatly improved the quality of this paper.

\section{REFERENCES}

1. Z. Artstein, Continuous dependence of solutions of Volterra integral equations, SIAM J. Math. Anal. 6 (1975), 446-456.

2. _ Continuous dependence on parameters: On the best possible results, J. Differential Equations 19 (1975), 214-225.

3. E. A. Coddington and N. Levinson, Theory of ordinary differential equations, McGraw-Hill, New York, 1955.

4. A. F. Filippov, Differential equations with discontinuous right-hand side, Mat. Sb. 51 (1960), 99-128 (Russian); Amer. Math. Soc. Transl. 42 (1964), 199-231.

5. I. I. Gihman, Concerning a theorem of N. N. Bogolyubov, Ukrainian Math. J. 4 (1952), 215-218.

6. S. Gutman, Evolutions governed by m-accretive plus compact operators, Nonlinear Anal. 7 (1983), 707-715.

7. _ Topological equivalence in the space of integrable vector-valued functions, Proc. Amer. Math. Soc. 93 (1985), 40-42.

8. L. Piccinini, G-convergence for ordinary differential equations, Proc. Internat. Meeting on Recent Methods in Nonlinear Analysis (E. DeGiorgi et al., eds.), Pitagora Editrice, Bologna, 1979.

9. E. Schechter, Existence and limits of Carathéodory-Martin evolutions, Nonlinear Anal. 5 (1981), 897-930.

10. __ One-sided continuous dependence of maximal solutions, J. Differential Equations 39 (1981), 413-425.

11. __ Perturbations of regularizing maximal monotone operators, Israel J. Math. 43 (1982), 49-61.

12. __ Evolution generated by semilinear dissipative plus compact operators, Amer. Math. Soc. Trans. 275 (1983), 297-308.

13. _ Necessary and sufficient conditions for convergence of temporally irregular evolutions, Nonlinear Anal. 8 (1984), 133-153.

14. G. I. Stassinopoulos and R. B. Vinter, Continuous dependence of solutions of a differential inclusion on the right hand side with applications to stability of optimal control problems, SIAM J. Control Optim. 17 (1979), 432-449.

15. $\mathrm{Z}$. Wu, The ordinary differential equations with discontinuous right members and the discontinuous solutions of the quasilinear partial differential equations, Sci. Sinica 13 (1964), 1901-1917.

Department of Mathematics, Western Kentucky University, Bowling Green, KenTUCKY 42101 